The hydrogen chloride was passed slowly into the benzene after running for fifteen minutes through the by-pass. At 9:42 A.M., Thursday, Jan. I5, all the zinc - both the c. p. wire and stick given me by Mr. Falk and the Schuchardt's stick zinc which I used orjginally - gave off gas. For a time gas came off faster and faster, the platinized zinc acting exactly like the other zinc except where the platinum coating protected it, and there no gas was evolved. No action was visible upon the magnesium: neither a tarnish nor gas evolution. The gradual formation of a protective coating upon all the zinc was seen, not a mere tarnish, but a thick white coat.

At I0:04-22 minutes from the start - there was still a decidedly brisk evolution of gas upon all the zinc.

At IO: IO -28 minutes - less gas was coming off.

At IO: $20-38$ minutes - very slow evolution of hydrogen. By shaking the flask about and getting a fresh zinc surface by abrasion of one piece of metal upon another, gas was again evolved upon all the zinc in spots, not uniformly, showing that the zinc ceased acting because of this protective coating and not because there was an insufficient quantity of water present to "ionize" the hydrochloric acid.

At IO: 30 gas still came slowly from all the zinc in spots, showing that where the coating was open the acid solution acted upon the zinc. The coating upon the zinc is thicker.

At Io: 40 gas coming from all the zinc at intervals of about 20 seconds between bubbles.

At I I: 00 minute bubbles from all the zinc at long intervals.

At $I: 45$ P.M. gas still coming slowly in tiny bubbles from all the zinc.

At 3: I 8 P.M. gas was evolved in small bubbles at long intervals on all the zinc. Confirmed by G. M. Wilcox of Armour Institute, and L. Kahlenberg.

At 5:00 P.M. all the zinc was covered with a thick even coating of white zinc chloride, which could not be dislodged by shaking the metals together, since no further action of zinc on the acid solution could be detected. 
During all this time a slow steady current of hydrogen chloride was passed into the benzene. The magnesium is not acted upon so far as could be detected by use of a large hand lense, either as to gas evolution or diminution of luster, after three days contact with the acid solution. In view of the important part which thin films play in the corrosion of metals it would be advisable to subject the magnesium and aluminum to the more delicate optical methods while in contact with this acid solution. So far as my observation goes the magnesium is not acted upon. The reason for this lack of action appears to me - in the absence of further experimental proof to the contrary - to be due to the same cause which underlies all differentiation of substances and which, the better to conceal our lack of knowledge, we call chemical affinity.

This action of hydrochloric acid gas dissolved in dry benzene upon dry zinc endured then five hours and thirty-six minutes, possibly longer. Dr. Remsen wished to get nascent hydrogen for reducing purposes in a solvent containing neither water, oxygen nor chlorine. While the hydrogen produced in my experiments may or may not serve this purpose, ${ }^{\mathrm{x}}$ the fact is estab. lished that we do get a gas evolved from the zinc; and within certain limits the dryer the materials the longer the action en. dures.

The following gentlemen were present at this last experiment:- Prof. W. W. Daniells, Prof. L. Kahlenberg, Prof. G. M. Wilcox, of Armour Institute, Mr. Shinn, Mr. Ferneckes, Mr. Huddle, and Mr. Brinsmaid. Prof. Lenher and Prof. C. F. Burgess examined the coating on the metals after the action had ceased.

Laboratory of Physical Chemistry, University of Wisconsin, Madison, Wis., Jan. 19, 1903 .

${ }^{1}$ I propose to try the reducing power of the hydrogen thus obtained at an early date. 


\section{ELECTROLYSIS OF WATER}

\section{BY W, R. WHITNEY}

The purpose of this article is to briefly discuss the continual electrolysis of water at voltages below the so-called decomposition value. On reading the article by Dr. Bigelow, ${ }^{\mathrm{T}}$ entitled "On the Passage of a Direct Current through an Electrolytic Cell", I was impressed with the idea that a strict application of the Nernst formula for E. M.F. of polarization would explain all the phenomena observed and described by him without the necessity of an introduction of any such corpuscular theory as he introduces. I am confident that this is the view held by others and is in accord with the theory as advanced by Helmholtz. Dr. Bigelow has repeated the early experiments of Helmholtz in which water, in closed vessels, is shown to continually conduct the current when the potential difference across the cell is only one volt. For visible decomposition, about I.7 volts is necessary, and this is approximately the value calculated by the erroneous process of considering the heat of the chemical reaction to be exactly equal to the electrical energy obtained from the reaction when generating a current, or equal to the electrical energy necessary to bring about the decomposition of water into its component gaseous products at atmospheric pressure. It seems to me that the recent advances in our electrochemical knowledge lead us to conclude that whenever an electromotive force is applied to a pair of electrodes in an electro. lyte, if its value is too small for visible electrolysis, there immediately occurs at least such an amount of real electrolysis as is capable of changing the poles into electrodes whose characteristic E. M. F. is equal and opposite to the impressed E. M. F. The earliest experiments on polarization point to this as a fact. In the case then of an aqueous solution, the electrodes at one

\footnotetext{
${ }^{1}$ Jour. Phys. Chem. 6, 603 (IgO2).
} 
volt impressed electromotive force are so charged with oxygen and hydrogen that they are to be considered as gas electrodes of low gas concentration or pressure. I see no reason for not accepting this conception as representing a condition to be expected. The gases could escape as bubbles only when the concentration had at least reached that value at which a gas pressure equal to that upon the liquid was reached, i. e., the atmospheric pressure. Helmholtz $z^{1}$ certainly recognized this principle when he drew the conclusion that with gas electrodes the electromotive force should be different for each different pressure of the gas. This he then expressed in the form,-

$$
\mathrm{A}-\mathrm{A}_{1}=\mathrm{C} \log p p_{p_{1}} .
$$

If these electrodes represent the gases at low pressure these gases should certainly dissolve in the water and tend to reach a condition of saturation corresponding to the above concentration. In an open vessel this might never be attained, owing to the continual escape of the gases into the air, where their partial pressures are low. As it is well known also that these gases when dissolved in the electrolyte are capable of depolarizing the electrodes, especially the hydrogen the oxygen electrode, a current at very low voltages seems a necessary result. It should depend largely on diffusion and convection, as the actually studied current very evidently does.

Considered from the point of view of energetics, there is also no transgression of a law. A molecule of water certainly could not be decomposed by an expenditure of less total energy than would be generated in its formation, nor is such an assumption necessary to the case in hand.

In the decomposition of water by the current in general, electrical energy and heat are both absorbed, but the quantitative relation between the two varies with the conditions. Moreover, at a given temperature it varies with the pressure so that at low pressures less electrical energy should be necessary than at high, although the total energy should be the same in all cases.

${ }^{1}$ Wied. Ann. 34, 737 (1888). 